\title{
Fuzzy Variable Order Extremum-Seeking Controller Design for Mobile Robots
}

\author{
O. ATAN
}

\begin{abstract}
In this paper, a fuzzy variable order extremumseeking control (FVO-ESC) system for a mobile robot are designed. Fractional order controllers have advantages in the control of nonlinear systems such as a wider area of stability and performance enhancement in the presence of noise. The main proposal of the paper is to increase the performance of the fractional order controller. So, a variable order controller was designed for a mobile robot, and a fuzzy logic controller was designed according to user experiences to tune the controller order. The proposed FVO-ESC approach has been validated the effects on nonlinear systems such as the mobile robot system. It has been put forward in the preliminary investigation that the order of the fractional order ESC controller affects the overshoot and time to reach the target. The results suggested that a variable-level controller would have better performance. The results show that the proposed FVO-ESC control approach provides optimum performance for mobile robot systems.
\end{abstract}

Index Terms - Mobile robots, Variable Order Systems, Extremum-Seeking Control.

\section{INTRODUCTION}

$\mathrm{T}$ HE EXTREMUM SEEKING control (ESC) method aims to meet nonlinear performance criteria of a system using the adaptive control method. The ESC method has been used in a lot of engineering systems such as nuclear reactors $[1,2]$, tracking the maximum power point of renewable energy systems [3-5], control of chemical systems [6-8], control of bioreactors [9-13], and mobile robots [14-18]. Because the ESC method has a lot of applications in the control of physical systems, researchers are interested in this control method. Especially due to increasing interest in autonomous vehicles and renewable energy systems, researchers have focused on the ESC control method. The following studies were focused recently: Atta realized a semi-global practical asymptotic stability analysis for phasor ESC with a nonlinear dynamic system [19]. The global extremum seeking point was designed and modelled using an asymptotic perturbed ESC and was applied to a photovoltaic system and fuel cell by Thounthong [20]. Wang improved an ESC algorithm without oscillation in

ÖZKAN ATAN, is with Department of Electrical-Electronic Engineering, Van Yüzüncü Y1l University, Van, Turkey, (e-mail: oatan@yyu.edu.tr).

(iD https://orcid.org/0000-0001-6443-9600

Manuscript received December 8, 2018; accepted January 31, 2019. DOI: $10.17694 /$ bajece. 492482 steady state for an antilock braking system, and the algorithm was compared with a sliding-mode-based ESC scheme for an antilock braking system [21]. Guay and Dochain considered real-time optimization of the ESC method and applied several example systems to show the effectiveness of their proposed system [22]. Nešić worked on dynamic feature and convergence analysis of ESC [23]. Hong and Li optimized ESC parameters using the particle swarm optimization (PSO) method, which guaranteed convergence [24]. These optimization methods and others aimed to decrease the convergence error and increase the speed of ESC for engineering applications $[7,12,14,15,25-30]$.

Malek and Chen, who introduced a different approach to the ESC method, used a fractional order integrator instead of the classical integrator in the ESC, and their work was found to outperform the classical ESC in simulation and application results [31, 32]. Because the fractional order control systems, which have non-integer order, have a wider region of stability than integer order control, fractional order control systems are shown to have better performance than classical integer control systems in the literature [33-36]. However, a significant problem in the fractional order control method is the determination of the parameters of the controller, and there are papers on determining the parameters based on intelligent systems in the literature [37-41]. The following intelligent methods for optimization of the fractional order control systems have been reported: Biswas et al. designed a fractional-order $\mathrm{PI}^{\lambda} \mathrm{D}^{\mu}$ controller using an improved differential evolution [37], Atan et al. used a fuzzy adaptive PSO algorithm for a fractional order PID controller and applied it to chaotic synchronization control [38]. Fractional order control parameters were optimized using PSO with improved dynamic parameter selection for a combined cycle power plant by Haji and Monje [39], and other researchers have used chaotic PSO for the optimization of the fuzzy fractional order control of a hybrid power system with renewable generation [40]. Another optimization method is based on the $\mathrm{ABC}$ algorithm for a fractional order controller [41]. These papers are focused on optimization of fractional order parameters, and constant values of the parameters are selected for each system. But when the parameters can be changed according to the system error, performance of the controller can be increased. It is known that the use of variable-order control systems improves the performance of the controller [42] as well as leading to successful results in variable order modelling of the systems [43].

In this paper, a variable order and fuzzy logic-based ESC method for a mobile robot model is proposed. Firstly, the 
analysis and numeric solution method for the modeling and simulation of variable order systems are presented, because variable order systems have different solution methods from fractional order systems. Because numerical solution techniques of variable order systems differ from the analysis of classic fractional order systems, Adams-Bashforth-Moulton analysis method, which is based on the Volterra integration method, have been used. With the stability analysis method, the order range of the system is defined in the stability area in this paper, and the other parameters are defined by the PSO method. In addition, the fractional controller parameters used for comparison are also optimized by the PSO method. To make a comparison with the proposed control system, the mobile robot system model is used as a test bench. The mobile robot can move on two axes to reach the target when controlled by the variable order ESC method and fractional order control. When the order of the ESC system is changed according to the error between the target and robot position, it is expected that the controller performance will increase.

This paper is organized as follows. The variable order system is formulated in Section II. The novel variable order extremum-seeking controller is presented in Section III. The stability of the mobile robot plant and variable order ESC are introduced in Section IV. The results are demonstrated in Section V, and finally, our conclusion is presented in Section VI.

\section{VARIABLE ORDER SYSTEMS}

\section{A. Fractional Order Calculus}

Despite the fact that fractional order systems have been introduced in the 17th century, which will lead to a useful paradox, the engineering applications on this topic are based on the 20th century [44]. These techniques are the GrunwaldLetnikov (GL) method (Eq. 1) and the Riemann-Liouville (RL) method (Eq. 2) [44, 45]. The GL equation is defined as the limit approximation as follows:

$$
a_{t}^{\alpha} f(t)=\operatorname{Lim}_{h \rightarrow \infty} \frac{1}{\Gamma(\alpha) h^{\alpha}} \sum_{k=0}^{[(t-a) / h]} \frac{\Gamma(\alpha+k)}{\Gamma(k+1)} f(x-k h)
$$

where $\Gamma$ is the gamma function. Another popular method is the RL method based on the continuous time function. The method is defined as:

$$
{ }_{a} D_{t}^{\alpha} f(t)=\frac{1}{\Gamma(m-\alpha)}\left(\frac{d}{d t}\right)^{m} \int_{a(t-\tau)}^{t-(m-\alpha)} d \tau
$$

where $\mathrm{m}$ is defined as $m-1<\alpha<m$. In recent years, many papers on fractional order systems analysis, which uses the Laplace transform method, have also appeared. GL approximation has been used for all the analysis in this paper.

\section{B. Analysis of Variable Order System.}

Several analysis methods have been proposed for variable order differential equations. Because the variable order differential equation is much more complex than the fractional order differential equations, a comprehensive study must be realized [46]. Although they have some negative features, researchers have focused on variable order systems because their success has been shown in several papers in the literature [47-50], and the researchers used generally Volterra integration model in the works. For variable order system analysis, Volterra integration can be used as follows:

$$
x(\mathrm{t})=\sum_{l=0}^{[\alpha]} x_{0}^{(\mathrm{l})} \frac{t^{l}}{l !}+\int_{0}^{t} \frac{(\mathrm{t}-\tau)^{\alpha(\tau)-1}}{\Gamma(\alpha(\tau))} f(\tau, \mathrm{x}(\tau)) \mathrm{d} \tau
$$

Voltera integration model is used continuous time model. For numerical solution methods, Adams-Bashforth-Moulton method is used. In this section, the Adams-Bashforth-Moulton predictor-corrector method is applied to implement the numerical solution of a function as:

$$
\begin{aligned}
& x_{k+1}=x_{0}+\frac{h^{q_{1}\left(\mathrm{t}_{\mathrm{k}+1}\right)}}{\Gamma\left(\mathrm{q}_{1}\left(\mathrm{t}_{\mathrm{k}+1}\right)+2\right)} f_{1}\left(\mathrm{x}_{k+1}^{p}\right) \ldots \\
& \ldots+\sum_{i=0}^{n} \frac{h^{q_{1}\left(\mathrm{t}_{\mathrm{k}+1}\right)} \gamma_{1, \mathrm{k}+1}}{\Gamma\left(\mathrm{q}_{1}\left(\mathrm{t}_{\mathrm{k}+1}\right)+2\right)} f_{1}\left(\mathrm{x}_{i}\right)
\end{aligned}
$$

where $x_{k+1}^{p}, t_{k+1}, \gamma_{i, \mathrm{k}+1}$, and $\beta_{1, \mathrm{k}+1}$ are given as follows:

$$
\begin{gathered}
x_{k+1}^{p}=x_{0}+\sum_{i=0}^{n} \frac{\beta_{1, \mathrm{k}+1}}{\Gamma\left(\mathrm{q}_{1}\left(\mathrm{t}_{k+1}\right)+2\right)} f_{1}\left(\mathrm{x}_{i}\right) \\
t_{k+1}=(n+1) h \\
\gamma_{i, \mathrm{k}+1}= \begin{cases}k^{q_{i}\left(\mathrm{t}_{k+1}\right)+1}-\left(\mathrm{k}-\mathrm{q}_{\mathrm{i}}\left(\mathrm{t}_{k+1}\right)\right)(k+1)^{q_{i}(\mathrm{t})} & i=0 \\
(k-\mathrm{i}-2)^{q_{i}\left(\mathrm{t}_{k+1}\right)+1}+(k-\mathrm{i})^{q_{i}\left(\mathrm{t}_{k+1}\right)+1} \cdots & 1 \leq i \leq k \\
\cdots-2(\mathrm{k}-\mathrm{i}+1)^{q_{i}\left(\mathrm{t}_{k+1}\right)+1} & i=k+1 \\
1 & \\
\beta_{i, \mathrm{k}+1}= & \frac{h^{q_{i}\left(\mathrm{t}_{k+1}\right)}}{q_{i}\left(\mathrm{t}_{k+1}\right)}\left((\mathrm{k}-\mathrm{i}+1)^{q_{i}\left(\mathrm{t}_{k+1}\right)+1}-(k-\mathrm{i})^{q_{i}\left(\mathrm{t}_{k+1}\right)+1}\right)\end{cases}
\end{gathered}
$$

where $\Gamma, \mathrm{h}$, and $\mathrm{k}$ parameters are given respectively gamma function, step range, and step. The numerical analysis method, which is used in this paper, is the most widely used in variable order calculus.

\section{VARIABLE ORDER EXTREMUM-SEEKING CONTROLLER}

\section{A. Classical Extremum-Seeking control}

Definition: ESC was firstly defined by Draper and $\mathrm{Li}$ in 1951. Although different algorithms for classical ESC have been presented, the sinusoidal perturbed ESC structure has drawn the most interest in the literature $[31,51]$. There are high- and low-pass filters in classical ESC, but the low-pass filter is eliminated to simplify the ESC. The form of a simplified ESC for a MIMO system is shown in Figure 1. As shown in Figure 1, periodic perturbation $(\sin (\omega t)$ and $\cos (\omega t))$ is used to estimate $\hat{x}$ and $\hat{y}$ There are high-pass filters in the scheme, and it is used to eliminate the DC signal in $\mathrm{x}$ and $\mathrm{y}$. In here, even if the filters do not directly affect the time taken to reach the target, they reduce the oscillation amplitude [31, 51]. The difference between the system points and extremum points in the classical ESC algorithm generates the amplitude of the sinus signal. In the system, in order to $\hat{\boldsymbol{x}}$ and $\hat{y}$ estimate approximately estimate the extremum $x^{*}$ and $y^{*}, J$ is defined as: 


$$
J=k_{a}-k_{x}\left(\hat{x}-x^{*}\right)^{2}-k_{y}\left(\hat{y}-y^{*}\right)^{2}
$$

where $k_{a}, k_{x}$, and $k_{y}$, which are adjusted approximation speed, and they are constant parameters.

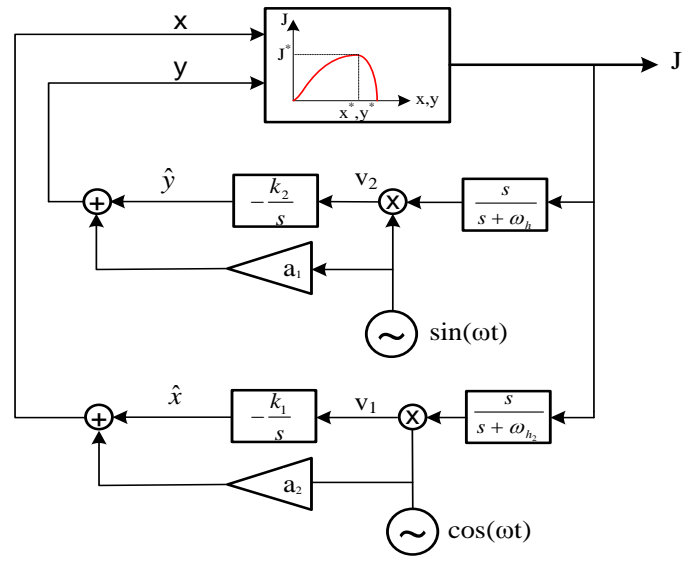

Fig. 1. Classical simplified extremum-seeking control (ESC)

$$
\begin{gathered}
v=\left[\begin{array}{l}
v_{1} \\
v_{2}
\end{array}\right]\left[\begin{array}{l}
\sin (\omega t) \cdot\left[J(x, y) * L^{-1}\left(\frac{s}{s+\omega_{h}}\right)\right] \\
\cos (\omega t) \cdot\left[J(x, y) * L^{-1}\left(\frac{s}{s+\omega_{h}}\right)\right]
\end{array}\right] \\
{\left[\begin{array}{l}
x \\
y
\end{array}\right]=\int\left[\begin{array}{cc}
-k_{1} & 0 \\
0 & -k_{2}
\end{array}\right]\left[\begin{array}{l}
v_{1} \\
v_{2}
\end{array}\right] d t+\left[\begin{array}{ll}
a_{1} & 0 \\
0 & a_{2}
\end{array}\right]\left[\begin{array}{c}
\sin (\omega t) \\
\cos (\omega t)
\end{array}\right]}
\end{gathered}
$$

The parameters $\left(k_{a}, k_{x}, k_{y}, a_{1}, a_{2}, \omega_{h}\right)$ in the control system are important for the performance of the system. So, for determination of the parameters, which must be tuned and calibrated adequately, ESC design rules are given in the literature $[31,52]$.

\section{B. Stability of Simplified ESC:}

For the stability of the ESC, the method given by Krstic in [53] is used in this paper. The model in Figure 1 is described by the equations:

$$
\begin{aligned}
& J=j^{*}+k_{x}\left(x-x^{*}\right)^{2}+k_{y}\left(x-x^{*}\right)^{2} \\
& x=a \cdot \cos (\omega t)-\frac{k}{s}\left(v_{1}\right) \\
& y=a \cdot \sin (\omega t)-\frac{k}{s}\left(v_{2}\right) \\
& v_{1}=a \cdot \cos (w t) \cdot\left[\frac{s}{s+\omega_{h}} * j(x, y)\right] \\
& v_{2}=\operatorname{arsin}(w t) \cdot\left[\frac{s}{s+\omega_{h}} * j(x, y)\right]
\end{aligned}
$$

When Lemmas 3.1-3.3 in [53] are applied to the system, it can be seen that the system has achieved stability conditions.

$$
\begin{aligned}
& v_{l}=a \cdot \frac{1}{2} \cdot\left(e^{i \omega t}+e^{-i \omega t}\right)\left[\frac{s}{s+\omega_{h}}\right] j(x, y) \\
& =a \cdot \frac{1}{2} \cdot L^{-1}\left[\begin{array}{c}
\frac{s}{(s-i \omega)\left(s+\omega_{h}\right)}+\ldots \\
\cdots \frac{s}{(s+i \omega)\left(s+\omega_{h}\right)}
\end{array}\right] j(x, y) \\
& =\frac{1}{2} a\left[\begin{array}{l}
\frac{1}{2} e^{-i t w}(1-i)+\frac{1}{4} e^{-t w}(a+2-i(2-a)) \ldots \\
\ldots+\frac{1}{4} a e^{i t w}(1+i)
\end{array}\right] j(x, y) \\
& v_{2}=a \cdot \frac{1}{i 2} \cdot\left(e^{i \omega t}-e^{-i \omega t}\right)\left[\frac{s}{s+\omega_{h}}\right] j(x, y) \\
& a \cdot \frac{1}{i 2} \cdot L^{-1}\left[\begin{array}{c}
\frac{s}{(s+i \omega)\left(s+\omega_{h}\right)} \ldots \\
\ldots-\frac{s}{(s-i \omega)\left(s+\omega_{h}\right)}
\end{array}\right] j(x, y) \\
& =\frac{1}{2} a\left[\begin{array}{l}
-\frac{1}{2} e^{-i t w}(1-i)-\frac{1}{4} e^{-t w}(a+2+i(a+2)) \ldots \\
\ldots+\frac{1}{4} a e^{i t w}(1-i)
\end{array}\right] j(x, y)
\end{aligned}
$$

$0<\omega$

$a<\infty$

\section{Design of Variable Order Extremum-Seeking Controller}

Fractional order ESC: A fractional order ESC, which is a combination of the ESC and fractional order systems, has been put forward by Malek and Chen [31]. The novelty in [31] is that the fractional order of the control system is defined with a fractional model, and the method has been used to increase the performance of the system. The block diagram of the fractional order ESC system is shown in Figure 2, and the effect of the fractional order on the control system is seen in Figure 3.

In Figure 3, it can be seen that the order of the fractional ESC affects the control performance. It is observed that the overshoot is increased but the setting time is decreased when the order $(\varphi)$ of the fractional ESC is decreased. Similarly, it is seen in Figure 3 that the optimum result is in the range of;

$$
0.9<\varphi<1.2
$$

If the order can change according to the error of the mobile robot position, the increase of the system performance can be observed. Therefore, in order to improve the performance of the mobile robot, the variable order ESC method is used in this paper.

\section{Control Order Changed by Fuzzy Logic}

Classical ESC, which is given in Figure 1, uses two low-pass filters and two integrators. In [31, 32], a fractional order integrator is used instead of a conventional integrator to increase the performance of the controller. The novelty in our work is that variable integrators are used instead of the classical integrators and fractional integrators, and the orders of the integrators are adjusted by fuzzy logic. 
A fuzzy variable order controller has been put forward by Pan and Xue [17], which changes the order of a variable order PI by the fuzzy logic method. The advantages of fuzzy adaptive control have been given in some papers $[54,55]$.

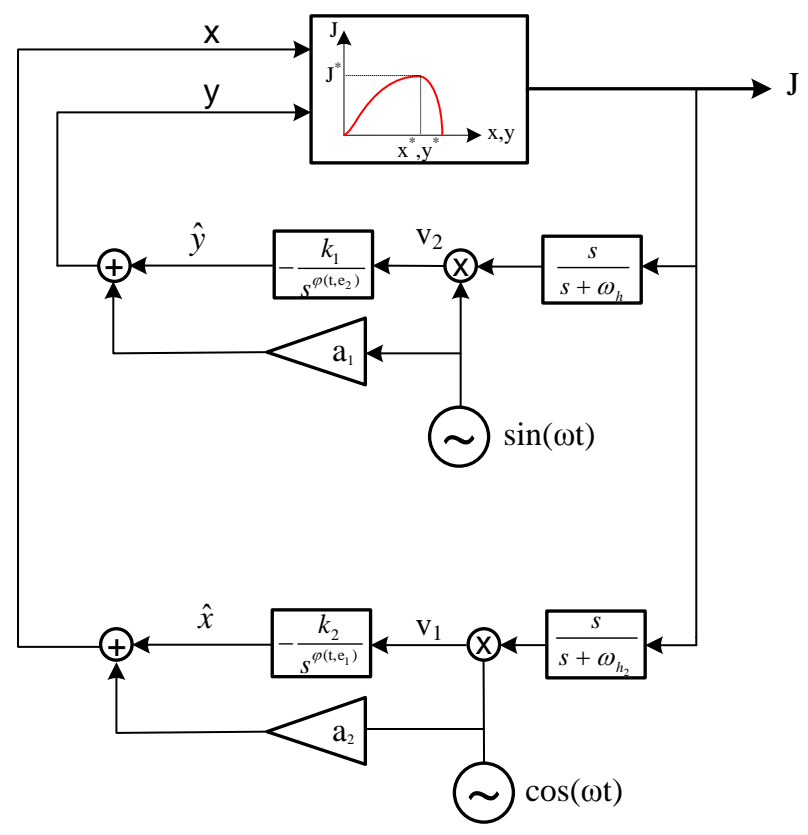

Fig. 2. Block diagram of the variable order ESC system

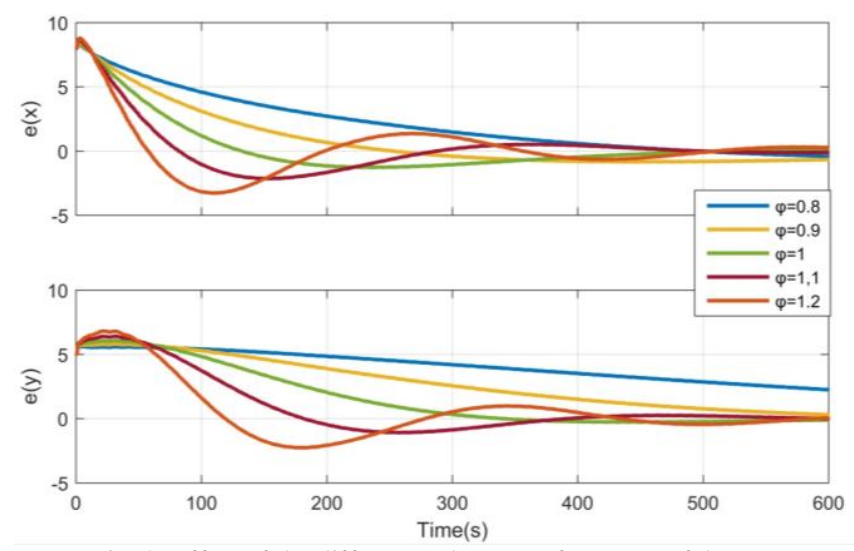

Fig. 3. Effect of the different order on performance of the system

These papers show effects of the adaptive control system such as overshoot, setting time, and the chattering phenomenon. In this paper, the orders of the variable-order ESC according to the rule bases in the fuzzy logic are changed in order to decrease the setting time. The fuzzy rules have two inputs and two outputs involved in the ESC. The system inputs are the $\mathrm{x}$ position error, the $\mathrm{y}$ position error, and their derivatives:

$$
\begin{aligned}
& e_{1}=x-x^{*} \\
& e_{2}=y-y^{*}
\end{aligned}
$$

where $e_{1,2}$ and $e_{1,2}$ are errors and the other system input is the derivative of the error. The variable order $\operatorname{ESC} \varphi_{1}$ and $\varphi_{2}$ are the outputs of the fuzzy system.

$$
\varphi_{1,2}=0.9+\varphi_{f u z z y_{1,2}}
$$

where $\varphi_{f u z z y_{1,2}}$ is calculated by fuzzy rules. The fuzzy system is designed using fuzzification, rule bases, and defuzzification.

The rules and membership functions are created according to the results from Figure 3. Rule bases are given in Table 1, and membership functions, which have mainly triangular shapes and five linguistic variables, are given in Figure 4. The linguistic variables are negative big (NB), negative $(\mathrm{N})$, zero $(\mathrm{Z})$, positive $(\mathrm{P})$, and positive big $(\mathrm{PB})$.

\begin{tabular}{|c|c|c|c|c|c|}
\hline$\dot{e}$ & $N B$ & $N$ & $Z$ & $P$ & $P B$ \\
\hline$N B$ & $N B$ & $N B$ & $N B$ & $N$ & $N$ \\
\hline$N$ & $N$ & $N$ & $N B$ & $N$ & $N$ \\
\hline$Z$ & $P B$ & $P$ & $Z$ & $N$ & $N B$ \\
\hline$P$ & $P B$ & $P B$ & $P B$ & $P$ & $P$ \\
\hline$P B$ & $P B$ & $P B$ & $P B$ & $P B$ & $P B$ \\
\hline
\end{tabular}

TABLE 1

FUZZY RULE BASE FOR $\varphi_{f u z z y_{1,2}}$
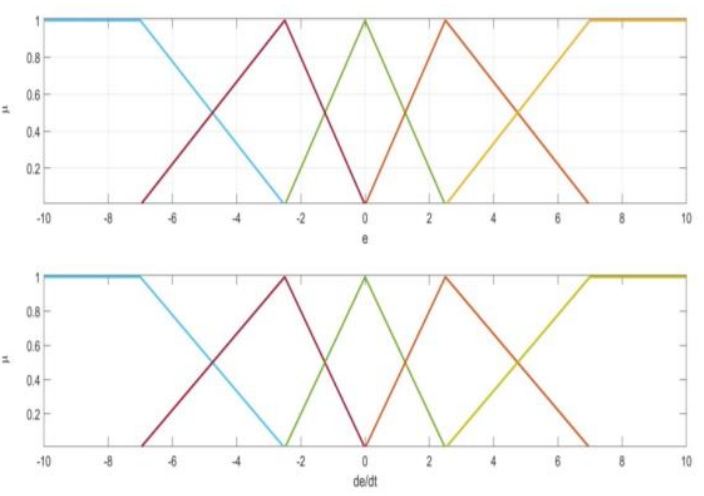

Fig. 4. Membership functions of $e_{1,2}$ and $\dot{e}_{1,2}$

\section{FUZZY VARIABLE ORDER EXTREMUM-SEEKING CONTROLLER FOR MOBILE ROBOT}

In this paper, in order to see the performance of the fuzzy variable order ESC and compare it with the other ESC methods, a mobile robot which is a $2 \mathrm{D}$ point mass vehicle, is employed (Figure 5). The system has two axes (x, y) and velocities $\left(\mathrm{v}_{\mathrm{x}}\right.$ and $\left.\mathrm{v}_{\mathrm{y}}\right)$ on the axes. The system dynamics are:

$$
\begin{aligned}
& \dot{x}=v e^{j \theta}=v_{x}+j v_{y} \\
& \dot{\theta}=\omega
\end{aligned}
$$

where $v_{x}$ and $v_{y}$ are the velocity on the $x$ and $y$ axes, and $\omega$ is the angular velocity.

In order to compare the performance of the fuzzy variable order ESC and classical ESC, both control methods use the same parameters in the control system.

\section{A. Fractional order ESC}

In this section, the performances of the mobile robot have been compared with the fractional order ESC. It is worth mentioning that the orders of the fractional order ESC were also defined by the performance index and the performance index has been chosen as $\mathrm{x}$ and $\mathrm{y}$ position errors. 


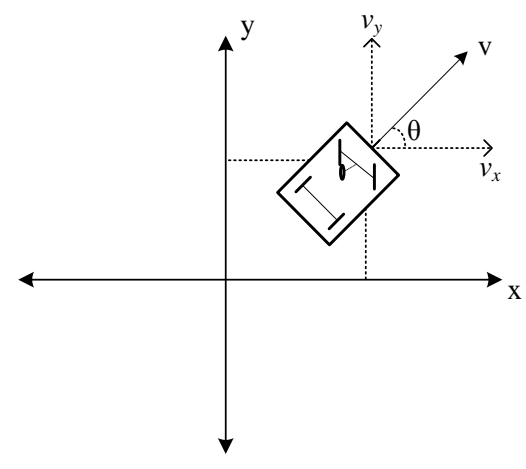

Fig. 5. A mobile robot on $2 \mathrm{D}$ points

The fractional order ESC and mobile robot parameters are given in Table 2, and to compare the performances of the mobile robot, the results of classical ESC and fractional order ESC for the same parameters are presented in Fig. 6.

TABLE 2

PARAMETERS OF THE CONTROLLER

\begin{tabular}{|l|l|l|l|l|l|l|}
\hline \multicolumn{7}{|c|}{ Control Parameters } \\
\hline$k_{l}$ & $k_{2}$ & $a_{1}$ & $a_{2}$ & $\omega_{1}$ & $\omega_{2}$ & $\Omega$ \\
\hline 1 & 1 & 0.5 & 0.5 & 1 & 1 & 20 \\
\hline
\end{tabular}
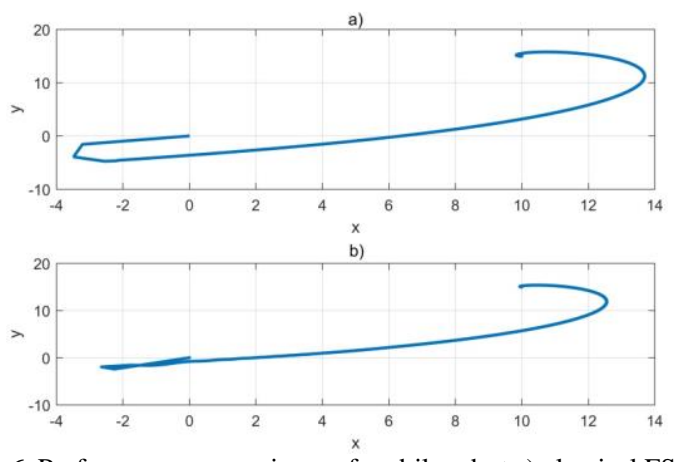

Fig. 6. Performance comparisons of mobile robot a) classical ESC b) fractional order ESC

It can be seen in Fig. 6 that the mobile robot employed to test the fractional-order ESC reached the destination by a shorter path and in a shorter time and clearly showed better performance than the classical ESC. From Figure 7, it can be clearly seen that the controller order affects the values of the overshoot and setting time. As the controller order increases, the overshoot increases, but this reduces the time it takes to reach a steady state. Based on this result, if the error has a small value, a small order is selected, and the order of the proposed controller increases in proportion with depending on the increase of the error. So, the performance indexes (setting time and overshoot) may have better values.
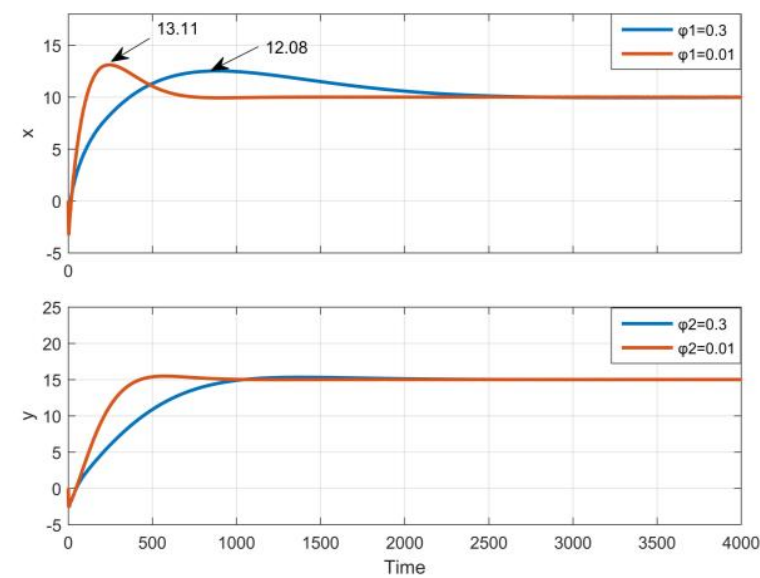

Fig. 7. Position errors of mobile robot for different fractional orders

\section{B. Fuzzy Variable ESC}

During the simulation, the mobile robot target vehicle follows the trajectory for $\mathrm{x}=15$ and $\mathrm{y}=10$ with the parameters set to the values given in Table 2. The value of $\mathbf{J}$ is initially high and then decreases during the simulation when using the proposed control method. Fractional order ESC has a more impressive result (overshoot and setting time) compared with classical ESC. The performance of the proposed control method shown in Figure 8, and the results have been compared with the results shown in Figure 6.

From Figure 8, it can be seen that fuzzy variable order ESC maintains satisfactory performance, namely a short rise time and less overshoot, when compared with classical ESC and fractional order ESC. For very complex systems such as mobile robot, the robot has better performance than classical ESC and fractional order ESC, as shown in Figure 9.

Changing of the fuzzy fractional order ESC, as shown in Figure 10, the system order is changed by the error. To compare with fractional order ESC and proposed controller, performance criteria such as overshoot and setting time are given in Table 3. It can be seen that the proposed controller has better performance than fractional order ESC. The overshoot decreased significantly $(5.8 \%)$ in the $\mathrm{x}$-axis, and relatively less in the $y$-axis. It is seen in Table 3 that there is a sitting time close to the average of both values in the fractional order controller.

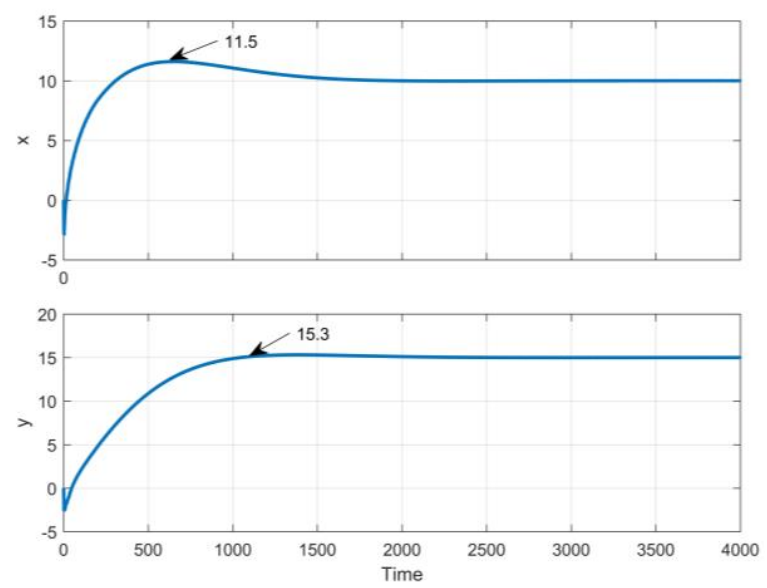

Fig. 8. Performance results of the proposed control method 


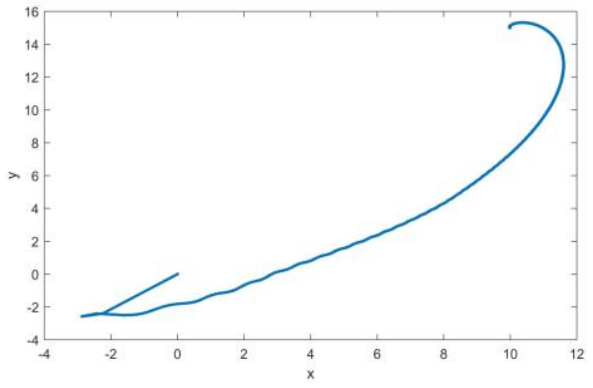

Fig. 9. Motion of the mobile robot on $2 \mathrm{D}$ points

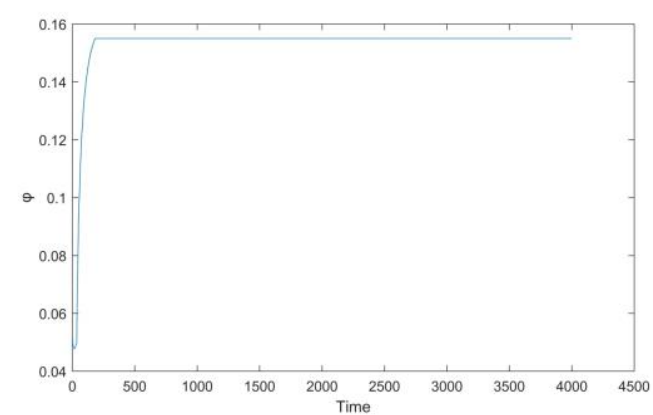

Fig. 10. The change of the system order

TABLE 3

OBTAINED RESULTS FROM PROPOSED CONTROLLER METHOD AND FRACTIONAL ORDER ESC

\begin{tabular}{|c|c|c|c|c|}
\hline \multirow{2}{*}{} & \multicolumn{3}{|c|}{ Control Method } \\
\cline { 2 - 5 } \multicolumn{2}{|c|}{ Fractional Order ESC } & Proposed Control \\
\hline \multicolumn{2}{|c|}{ Parameters } & $\varphi=0.01$ & $\varphi=0.3$ & \\
\hline \multirow{2}{*}{$x$} & Overshoot & 3.11 & 2.08 & 1.5 \\
\cline { 2 - 5 } & Setting time & $720 s$ & $2400 s$ & $1540 s$ \\
\hline \multirow{2}{*}{$y$} & Overshoot & 0.45 & 0.5 & 0.3 \\
\cline { 2 - 5 } & Setting time & $934 s$ & $2110 s$ & $1452 s$ \\
\hline
\end{tabular}

\section{CONCLUSION}

This paper proposes variable order ESC using fuzzy logic for a mobile robot. The order of the ESC controller is based on fractional calculus, adaptively adjusted with fuzzy logic. The performance analyses based on the overshoot and rise time show that the proposed variable order of the controller can provide improvements in performance improvement compared to the fractional order ESC algorithm. The fractional order ESC model was first designed by Malek and Chen [31], [32], who proved that ESC provides good performance against noise and uncertainties. The controller order affects the overshoot and the setting time for the mobile robot have shown, and the approach provides a remarkable performance enhancement, because a decrease in the value of the order causes an increase in the amount of overtaking but gives a better result in terms of setting time. So, if the controller order according to the tracking error can be changed, it will show better performance than classical fractional order control. The simulation results for a mobile robot in this paper justify the performance enhancement.

\section{REFERENCES}

[1] D. Carnevale, A. Astolfi, C. Centioli, S. Podda, V. Vitale, and L. Zaccarian, "A new extremum seeking technique and its application to maximize RF heating on FTU," Fusion Eng. Des., vol. 84, no. 2-6, pp. 554-558, Jun. 2009.

[2] Y. Ou et al., "Design and simulation of extremum-seeking open-loop optimal control of current profile in the DIII-D tokamak," Plasma Phys. Control. Fusion, vol. 50, no. 11, p. 115001, Nov. 2008.

[3] U. Ciri, M. A. Rotea, and S. Leonardi, "Model-free control of wind farms: A comparative study between individual and coordinated extremum seeking," Renew. Energy, vol. 113, pp. 1033-1045, 2017.

[4] B. Hu, Y. Li, B. Mu, S. Wang, J. E. Seem, and F. Cao, "Extremum seeking control for efficient operation of hybrid ground source heat pump system," Renew. Energy, vol. 86, pp. 332-346, 2016.

[5] N. Bizon, "Global Maximum Power Point Tracking (GMPPT) of Photovoltaic array using the Extremum Seeking Control (ESC): A review and a new GMPPT ESC scheme," Renew. Sustain. Energy Rev., vol. 57, pp. 524-539, 2016.

[6] H. C. Lee, S. Kim, J. P. Heo, D. H. Kim, and J. Lee, "Wiener Model and Extremum Seeking Control for a CO Preferential Oxidation Reactor with the $\mathrm{CuO}-\mathrm{CeO} 2$ catalyst," IFAC-PapersOnLine, vol. 48, no. 8, pp. 574-579, 2015.

[7] G. Lara-Cisneros, R. Aguilar-López, and R. Femat, "On the dynamic optimization of methane production in anaerobic digestion via extremum-seeking control approach," Comput. Chem. Eng., vol. 75, pp. 49-59, 2015.

[8] D. Krishnamoorthy, A. Pavlov, and Q. Li, "Robust Extremum Seeking Control with application to Gas Lifted Oil Wells," IFAC-PapersOnLine, vol. 49, no. 13, pp. 205-210, 2016.

[9] P. Cougnon, D. Dochain, M. Guay, and M. Perrier, "On-line optimization of fed-batch bioreactors by adaptive extremum seeking control," IFAC Proc. Vol., vol. 43, no. 6, pp. 108-113, 2010.

[10] M. Titica, D. Dochain, and M. Guay, "Real-Time Optimization of FedBatch Bioreactors via Adaptive Extremum-Seeking Control," Chem. Eng. Res. Des., vol. 81, no. 9, pp. 1289-1295, 2003.

[11] P. Cougnon, D. Dochain, M. Guay, and M. Perrier, "On-line optimization of fedbatch bioreactors by adaptive extremum seeking control," J. Process Control, vol. 21, no. 10, pp. 1526-1532, 2011.

[12] A. Vargas, J. A. Moreno, and A. Vande Wouwer, "Super-twisting estimation of a virtual output for extremum-seeking output feedback control of bioreactors," J. Process Control, vol. 35, pp. 41-49, 2015.

[13] O. Trollberg and E. W. Jacobsen, "Greedy Extremum Seeking Control with Applications to Biochemical Processes," IFAC-PapersOnLine, vol. 49, no. 7, pp. 109-114, 2016.

[14] Y. Zhang, O. Makarenkov, and N. Gans, "Extremum seeking control of a nonholonomic system with sensor constraints," 2016.

[15] V. Koropouli, A. Gusrialdi, S. Hirche, and D. Lee, "An extremumseeking control approach for constrained robotic motion tasks," Control Eng. Pract., vol. 52, pp. 1-14, 2016.

[16] A. S. Matveev, M. C. Hoy, and A. V. Savkin, "3D environmental extremum seeking navigation of a nonholonomic mobile robot," Automatica, vol. 50, no. 7, pp. 1802-1815, 2014.

[17] Y. Tian, N. Sarkar, Y. Tian, and N. Sarkar, "Control of a Mobile Robot Subject to Wheel Slip," J Intell Robot Syst, vol. 74, pp. 915-929, 2014.

[18] C. Li, Z. Qu, and M. A. Weitnauer, "Distributed extremum seeking and formation control for nonholonomic mobile network," Syst. Control Lett., vol. 75, pp. 27-34, 2015.

[19] K. T. Atta, A. Johansson, and T. Gustafsson, "On the stability analysis of phasor and classic extremum seeking control," Syst. Control Lett., vol. 91, pp. 55-62, 2016.

[20] P. Thounthong, M. Raducu, and L. M. Constantinescu, "Designing and modelling of the asymptotic perturbed extremum seeking control scheme for tracking the global extreme," Int. J. Hydrogen Energy, vol. 42, no. 28 , pp. 17632-17644, 2017.

[21] L. Wang, S. Chen, and K. Ma, "On stability and application of extremum seeking control without steady-state oscillation," Automatica, vol. 68, pp. 18-26, 2016.

[22] M. Guay and D. Dochain, "A time-varying extremum-seeking control approach," Automatica, vol. 51, pp. 356-363, Jan. 2015.

[23] D. Nešić, "Extremum Seeking Control: Convergence Analysis," Eur. J. Control, vol. 15, no. 3-4, pp. 331-347, Jan. 2009. 
[24] C. Hong and K. Li, "Swarm intelligence-based extremum seeking control," Expert Syst. Appl., vol. 38, no. 12, pp. 14852-14860, Nov. 2011.

[25] M. Haring, N. van de Wouw, and D. Nešić, "Extremum-seeking control for nonlinear systems with periodic steady-state outputs," 2013.

[26] C. Yin, B. Stark, and S. Zhong, "Adaptive minimum energy cognitive lighting control: Integer order vs fractional order strategies in sliding model-based extremum seeking," Mechatronics, vol. 23, no. 7, pp. 863872, 2013.

[27] K. T. Atta, A. Johansson, and T. Gustafsson, "Extremum seeking control based on phasor estimation," Syst. Control Lett., vol. 85, pp. 37-45, 2015

[28] S. Z. Khong, D. Nešić, and M. Krstić, "Iterative learning control based on extremum seeking," 2016.

[29] B. Mu, Y. Li, J. M. House, and T. I. Salsbury, "Experimental evaluation of anti-windup extremum seeking control for airside economizers," Control Eng. Pract., vol. 50, pp. 37-47, 2016.

[30] C. Yin, S. Dadras, X. Huang, J. Mei, H. Malek, and Y. Cheng, "Energysaving control strategy for lighting system based on multivariate extremum seeking with Newton algorithm," Energy Convers. Manag., vol. 142, pp. 504-522, 2017.

[31] H. Malek and Y. Chen, "Fractional Order Extremum Seeking Control: Performance and Stability Analysis," IEEE/ASME Trans. Mechatronics, vol. 21, no. 3, pp. 1620-1628, Jun. 2016.

[32] H. Malek, S. Dadras, and Y. Chen, "Performance analysis of fractional order extremum seeking control," ISA Trans., vol. 63, pp. 281-287, 2016.

[33] C. A. Monje, Y. Chen, B. M. Vinagre, D. Xue, and V. Feliu, Fractionalorder Systems and Controls. London: Springer London, 2010.

[34] S. E. Hamamci, "An Algorithm for Stabilization of Fractional-Order Time Delay Systems Using Fractional-Order PID Controllers," IEEE Trans. Automat. Contr., vol. 52, no. 10, pp. 1964-1969, Oct. 2007.

[35] Y. Chen, I. Petras, and D. Xue, "Fractional order control - A tutorial," in 2009 American Control Conference, 2009, pp. 1397-1411.

[36] M. Beschi, F. Padula, and A. Visioli, "The generalised isodamping approach for robust fractional PID controllers design,” Int. J. Control, vol. 90, no. 6, pp. 1157-1164, Jun. 2017.

[37] A. Biswas, S. Das, A. Abraham, and S. Dasgupta, "Design of fractionalorder PI $\lambda \mathrm{D} \mu$ controllers with an improved differential evolution," Eng. Appl. Artif. Intell., vol. 22, no. 2, pp. 343-350, Mar. 2009.

[38] O. Atan, M. Turk, and R. Tuntas, "Fractional Order Controller Design for Fractional Order Chaotic Synchronization,” Int. J. Nat. Eng. Sci., vol. 7, no. 1, pp. 71-77, 2013.

[39] V. Haji Haji and C. A. Monje, "Fractional order fuzzy-PID control of a combined cycle power plant using Particle Swarm Optimization algorithm with an improved dynamic parameters selection," Appl. Soft Comput., vol. 58, pp. 256-264, Sep. 2017.

[40] I. Pan and S. Das, "Fractional order fuzzy control of hybrid power system with renewable generation using chaotic PSO," ISA Trans., vol. 62, pp. 19-29, May 2016.

[41] H. Senberber and A. Bagis, "Fractional PID controller design for fractional order systems using ABC algorithm," in 2017 Electronics, 2017, pp. 1-7.

[42] L. Liu, F. Pan, and D. Xue, "Variable-order fuzzy fractional PID controller," ISA Trans., vol. 55, pp. 227-233, Mar. 2015.

[43] S. Ma, Y. Xu, and W. Yue, "Numerical Solutions of a Variable-Order Fractional Financial System,” J. Appl. Math., vol. 2012, pp. 1-14, Sep. 2012.
[44] I. Podlubny, Fractional differential equations: an introduction to fractional derivatives, fractional differential equations, to methods of their solution and some of their applications. Academic Press, 1999.

[45] S. M. Miller and R. Bertram, An Introduction to the Fractional Calculus and Fractional Differential EquationsNo Title. USA: Wiley, 1993.

[46] S. Ma, Y. Xu, and W. Yue, "Numerical Solutions of a Variable-Order Fractional Financial System," J. Appl. Math., vol. 2012, pp. 1-14, 2012.

[47] D. Sierociuk and M. Macias, "Comparison of variable fractional order PID controller for different types of variable order derivatives," in Proceedings of the 14th International Carpathian Control Conference (ICCC), 2013, pp. 334-339.

[48] P. Ostalczyk, D. Brzezinski, P. Duch, M. Łaski, and D. Sankowski, "The variable, fractional-order discrete-time PD controller in the IISv1.3 robot arm control," Open Phys., vol. 11, no. 6, pp. 750-759, Jan. 2013.

[49] P. Ostalczyk, "Variable-, fractional-order discrete PID controllers," in 2012 17th International Conference on Methods \& Models in Automation \& Robotics (MMAR), 2012, pp. 534-539.

[50] A. Razminia, A. F. Dizaji, and V. J. Majd, "Solution existence for nonautonomous variable-order fractional differential equations," Math. Comput. Model., vol. 55, no. 3-4, pp. 1106-1117, Feb. 2012.

[51] Q. Zheng and Z. Gao, 2010 29th Chinese Control Conference. I E E E, 2010.

[52] K. B. Ariyur and M. Krstić, Real-Time Optimization by ExtremumSeeking Control, vol. 0, no. 0. 2003.

[53] M. Krstiã, "Performance improvement and limitations in extremum seeking control," Syst. Control Lett., vol. 39, pp. 313-326, 2000.

[54] Y. Li, S. Sui, and S. Tong, "Adaptive Fuzzy Control Design for Stochastic Nonlinear Switched Systems with Arbitrary Switchings and Unmodeled Dynamics," IEEE Trans. Cybern., pp. 1-12, 2016.

[55] A. Boulkroune, "A fuzzy adaptive control approach for nonlinear systems with unknown control gain sign," Neurocomputing, vol. 179, pp. 318-325, Feb. 2016.

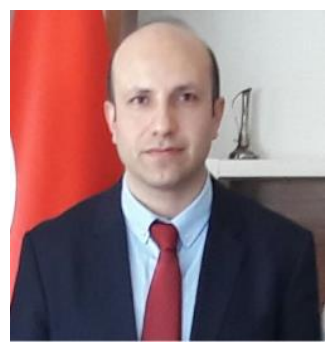

Özkan ATAN He received the M.Sc. and Ph.D. degrees in Electrical and Electronics Engineering from Yüzüncü Yll University (Van, Turkey) and Firat University (Elazığ, Turkey) in 2007 and 2014, respectively. $\mathrm{He}$ is an Assistant Professor in the Electrical and Electronics Engineering Department of Yüzüncü Yıl University. He works on control systems, chaotic \& nonlinear systems, fractional order systems. 